\title{
Etude de la sensibilité d'Enchytraeus variatus (Oligochaeta, Enchytraeidae) à certains sels de métaux lourds en vue de son utilisation comme organisme test
}

\author{
C. Guérin! 1 \\ N. Gianil \\ M. Sirel
}

Mots-clés : Enchytraeus, biologie, métaux lourds, toxicité, organisme test.

$E$. variatus est une espèce de petite taille facile à élever au laboratoire ; elle peut se reproduire par voie asexuée (fragmentation). L'influence de certains sels de métaux lourds sur la mortalité, le taux de fragmentation et la croissance des populations ont été étudiés afin d'établir les possibilités d'utilisation de cette espèce amphibie pour tester la toxicité des sédiments aquatiques. Les sels de $\mathrm{Cd}, \mathrm{Cu}$ et $\mathrm{Zn}$ provoquent une mortalité proportionnelle à leur concentration. Le zinc est le métal le plus nocif ; le plomb n'entraîne pas de mortalité significative à court terme ( 72 heures). Les sels de métaux utilisés modifient de façon variable le taux de fragmentation et de croissance des populations. La fragmentation complique l'interprétation des résultats des tests auxquels cette espèce est soumise. De ce fait, $E$. variatus ne semble pas être un bon candidat comme organisme test, comme le sont d'autres espèces du même genre.

A study of the sensitivity of Enchytraeus variatus (Oligochaeta, Enchytraeidae) to certain salts of heavy metals in relation to its use as a test organism

Keywords : Enchytraeus, biologie, heavy metals, toxicity, test organism

$E$. variatus is a small species that is easy to rear in the laboratory; it is able to reproduce asexually (fragmentation). The influence of certain salts of heavy metals on the mortality, fragmentation rate and growth of populations has been studied in order to establish the possibilities of using this amphibious species to test the toxicity of aquatic sediments. Salts of $\mathrm{Cd}, \mathrm{Cu}$ and $\mathrm{Zn}$ produced a mortality proportional to their concentration. Zinc is the most harmful metal ; lead did not cause a significant mortality in a short time period ( 72 hours). The metal salts used in the experiments modified in a variable way the fragmentation rate and growth of the populations. Fragmentation complicates the interpretation of the results of the tests used on this species. Hence, $E$. variatus does not seem to be suitable as a test organism, as are other species of the same genus.

\section{Introduction}

Les Enchytraeidae ont rëcemment été utilisés pour tester la toxicité de certaines substances dans les sols (Kaufman 1975, Bethke-Beilfuss 1986, Römbke \& Knacker 1989, Westheide \& Bethke-Beilfuss 1991) ou dans les milieux aquatiques (Römbke \& Knacker op. cit.). Ce sont généralement des espèces du genre Enchytraeus qui ont été employées.

1. Laboratoire d'Hydrobiologie, Université Paul Sabatier, URA CNRS 695, 118, route de Narbonne, 31062 Toulouse Cedex, France.
Les caractéristiques d'une nouvelle espèce, Enchytraeus variatus Bouguenec \& Giani, 1987, ont fait de cette forme un organisme test potentiel. En effet, $E$. variatus est un ver de petite taille $(6$ à $11,5 \mathrm{~mm}$, 25 à 31 segments), aisé à élever. Son temps de génération est très court, 26 jours en moyenne d'œuf à cuf ; il peut se reproduire asexuellement, parfois exclusivement, par fragmentation (Bouguenec \& Giani 1989). Cette espèce terrestre ou amphibie pourrait permettre de tester, en laboratoire, la toxicité de sédiments prélevés dans le milieu naturel et utilisés comme substrat d'élevage, en l'absence d'eau surnageante. 
Dans cette optique, nous avons réalisé une analyse préliminaire, destinée à évaluer la sensibilité de cette espèce. Cette étude est basée sur l'observation en laboratoire des effets de sels de métaux lourds (plomb, cuivre, zinc et cadmium), utilisés seuls ou associés deux par deux, sur la mortalité, la fragmentation et la croissance des populations de cet Enchytraeidae. L'influence du plomb et celle du cadmium ont été précisées en fonction de la température, de la teneur en matière organique du substrat et du $\mathrm{pH}$.

\section{Matériel et méthodes}

Les vers ont été élevés selon la méthode préconisée par Bouguenec (1987). Les expériences ont été effectuées dans des boîtes en plastique (dimensions : $4 \times 5 \times 1 \mathrm{~cm}$ ) dont le fond était recouvert par 1 gramme de terreau de tourbe de sphaigne et de carex (NF U44-551) humidifié à l'aide de $3 \mathrm{ml}$ de solution du (des) sel(s) métallique(s) à tester (Tableaux 1 et 2) ou d'eau du robinet pour les témoins.

Tableau 1. Concentrations (en $\mathrm{mg} / \mathrm{g}$ de poids sec) des métaux utilisés lors des tests.

Table 1 . Concentrations (in $\mathrm{mg} / \mathrm{g}$ dry weight) of metals used for the tests.

\begin{tabular}{|c|c|c|c|c|c|}
\hline $\begin{array}{l}\mathrm{Cd} \\
\mathrm{Cu} \\
\mathrm{Zn} \\
\mathrm{Pb}\end{array}$ & $\begin{array}{r}14,7 \\
11,3 \\
11,5 \\
3,0\end{array}$ & $\begin{array}{l}25,7 \\
34,0 \\
22,9 \\
24,0\end{array}$ & $\begin{array}{r}36,7 \\
56,7 \\
34,4 \\
400,0\end{array}$ & $\begin{array}{r}55,2 \\
70,8 \\
45,8 \\
801,3\end{array}$ & $\begin{array}{r}73,6 \\
85,0 \\
57,3 \\
1600,0\end{array}$ \\
\hline
\end{tabular}

Au début de chacune des expériences, 10 vers adultes, non sexuellement matures, ont été introduits dans les boîtes. Chaque essai a fait l'objet de 3 réplicats accompagnés de 3 témoins. Tous les tests ont été réalisés à l'obscurité. Les paramètres dont nous n'avons pas étudié l'influence sont restés constants $\left(\mathrm{pH}=5,8\right.$; température $=20^{\circ} \mathrm{C}$; teneur en matière organique $=54 \%$ ).

Le cadmium, le cuivre et le zinc ont été utilisés sous forme de chlorures en solution aqueuse et le plomb sous forme de citrate et de carbonate. Pour étudier les effets d'éventuelles synergies, les métaux ont été associés deux par deux (Tableau 2).

L'influence des facteurs environnementaux a été étudiée sur le plomb à la concentration de $15,6 \mathrm{mg} / \mathrm{l}$, et le cadmium à $3,5 \mathrm{mg} / \mathrm{l}$, utilisés sous forme de solution de $\mathrm{Pb}(\mathrm{NO} 3)_{2}$, et de $\mathrm{CdCl}_{2}$. L'influence de la température a été étudiée à 10,20 et $30^{\circ} \mathrm{C}$ et celle du $\mathrm{pH}$ du substrat pour des valeurs de $5,5,7$ et 8,5 . L'ajout de sable calciné au terreau a permis d'obtenir des substrats à $54 \%, 27 \%$ et $13,5 \%$ de teneur en matière organique.

Les expériences sur les effets à court terme ont duré 72 heures ; des essais préliminaires ont montré que, comme l'avaient noté Römbke \& Knacker (1989), les effets observés après 96 heures ne diffèrent pas significativement de ceux observés après 72 heures. Les individus et les fragments ont été dénombrés toutes les 24 heures.

Les expériences sur les effets à moyen terme ont duré 14 et 39 jours. Deux concentrations en plomb et en cadmium ont été utilisées : $1,2 \mathrm{mg} / \mathrm{g}$ PS puis 0,5 pour le $\mathrm{Pb}, 0,3 \mathrm{mg} / \mathrm{g}$ PS puis 0,1 pour le $\mathrm{Cd}$.

Les principaux paramètres étudiés sont :

Tableau 2. Effets des associations entre les métaux (concentrations en $\mathrm{mg} / \mathrm{g}$ de poids sec) sur la mortalité d' $E$. variatus : S : synergie ; $\mathrm{A}$ : antagonisme ; $\mathrm{R}$ : rien, aucun effet. Dans la partie inférieure du tableau figurent les codes utilisés pour identifier les diverses associations.

Table 2. Effects of associations between the metals (concentrations in $\mathrm{mg} / \mathrm{g}$ dry weight) on the mortality of $E$. variatus : $\mathrm{S}:$ synergy ; $\mathrm{A}$ : antagonism ; $\mathrm{R}$ : nothing, no effect. The codes used to identify the different associations are figured in the lower part of the table.

\begin{tabular}{|c|c|c|c|c|c|c|c|c|c|}
\hline \multirow{2}{*}{\multicolumn{2}{|c|}{ Concentrations }} & \multicolumn{2}{|c|}{ CADMIUM } & \multicolumn{2}{|c|}{ CUIVRE } & \multicolumn{2}{|r|}{ ZINC } & \multicolumn{2}{|c|}{ PLOMB } \\
\hline & & 14,7 & 36,7 & 11,3 & 34 & 11,5 & 22,9 & & 24 \\
\hline CADMIUM & $\begin{array}{l}14,7 \\
36,7\end{array}$ & & & $\begin{array}{l}\mathbf{S} \\
\mathbf{S}\end{array}$ & $\begin{array}{l}\mathbf{S} \\
\mathbf{S}\end{array}$ & $\begin{array}{l}\mathbf{R}^{1} \\
\mathbf{R}\end{array}$ & $\begin{array}{l}\mathbf{S} \\
\mathbf{S}\end{array}$ & $\begin{array}{l}\mathbf{R} \\
\mathbf{S}\end{array}$ & $\begin{array}{l}\mathbf{R} \\
\mathbf{S}\end{array}$ \\
\hline CUIVRE & $\begin{array}{l}11 ; 3 \\
34\end{array}$ & $\begin{array}{l}\text { CC1 } \\
\text { CC } 3\end{array}$ & $\begin{array}{l}\mathrm{CC} 2 \\
\mathrm{CC} 4\end{array}$ & & & $\begin{array}{l}\mathbf{R} \\
\mathbf{R}\end{array}$ & $\begin{array}{l}\mathbf{R} \\
\mathbf{R}\end{array}$ & $\begin{array}{l}\mathbf{R} \\
\mathbf{R}\end{array}$ & $\begin{array}{l}\mathbf{R} \\
\mathbf{R}\end{array}$ \\
\hline ZINC & $\begin{array}{l}11,5 \\
22,9\end{array}$ & $\begin{array}{l}\text { CZ1 } \\
\text { CZ3 }\end{array}$ & $\begin{array}{l}\text { CZ2 } \\
\text { CZ4 }\end{array}$ & $\begin{array}{l}\mathrm{zC1} \\
\mathrm{zC} 3\end{array}$ & $\begin{array}{l}\mathrm{ZC2} \\
\mathrm{ZC4}\end{array}$ & & & $\begin{array}{l}\text { A } \\
\text { A }\end{array}$ & $\begin{array}{l}\text { A } \\
\text { A }\end{array}$ \\
\hline PLOMB & $\begin{array}{c}3 \\
24\end{array}$ & $\begin{array}{l}\text { PC1 } \\
\text { PC3 }\end{array}$ & $\begin{array}{l}\text { PC2 } \\
\text { PC4 }\end{array}$ & $\begin{array}{l}\text { CP1 } \\
\text { CP3 }\end{array}$ & $\begin{array}{l}\text { CP2 } \\
\text { CP4 }\end{array}$ & $\begin{array}{l}\text { PZ1 } \\
\text { PZ3 }\end{array}$ & $\begin{array}{l}\text { PZ2 } \\
\text { PZ4 }\end{array}$ & . & \\
\hline
\end{tabular}


1) le taux de mortalité et son corrolaire, la DL-50 ; ils ont été déterminés pour les adultes et les jeunes individus (fragments) qui peuvent avoir une sensibilité différente (Römbke \& Knacker 1989) ;

2) les effets sublétaux sur la fragmentation (exprimée par le taux de fragmentation) et sur la croissance de la population (exprimée par le taux d'accroissement).

\section{Résultats}
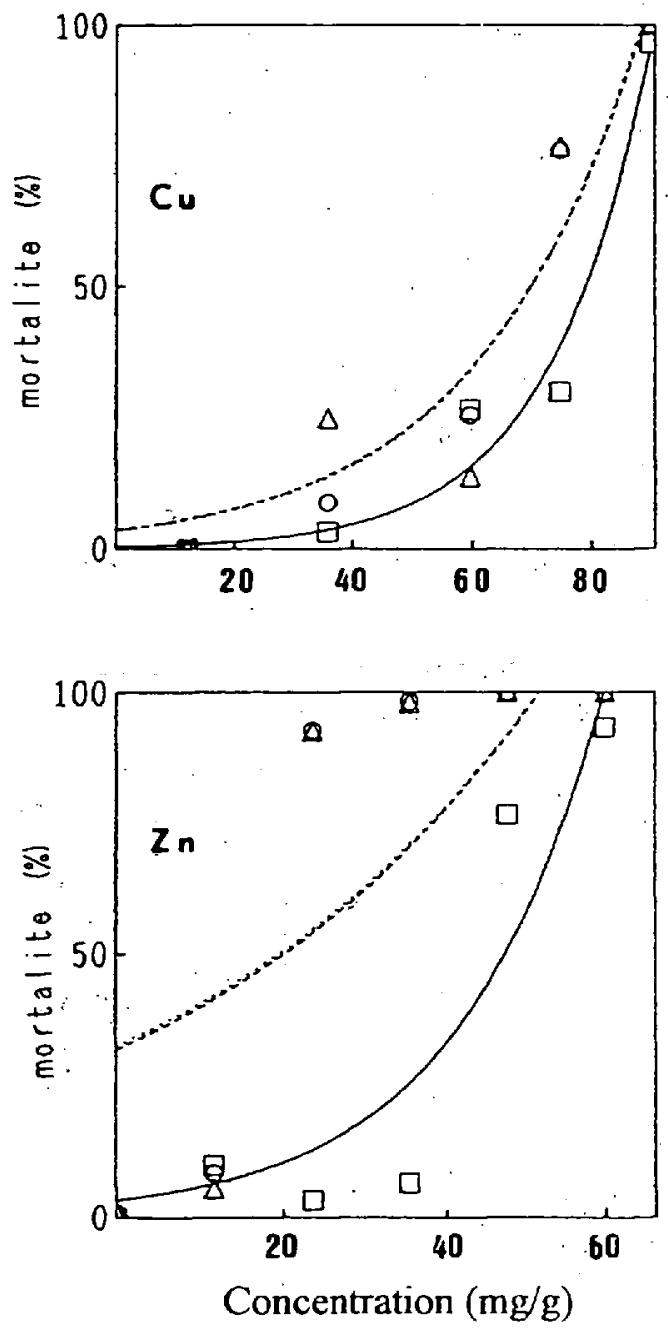

$\longrightarrow$ - adultes; - - - - - - jeunes;

\subsection{Effets à court terme}

\section{Mortalité}

Dans le cas du cadmium, du zinc et du cuivre la mortalité de la population totale (adultes + fragments) est une fonction plus ou moins expónentielle de la concentration (Fig. 1) ; elle est complète pour la plus forte concentration testée de cadmium et de cuivre et pour les deux plus fortes. de zinc. En présence de plomb, la mortalité d'E. variatus est faible (Fig. $1 \mathrm{~Pb}$ ).
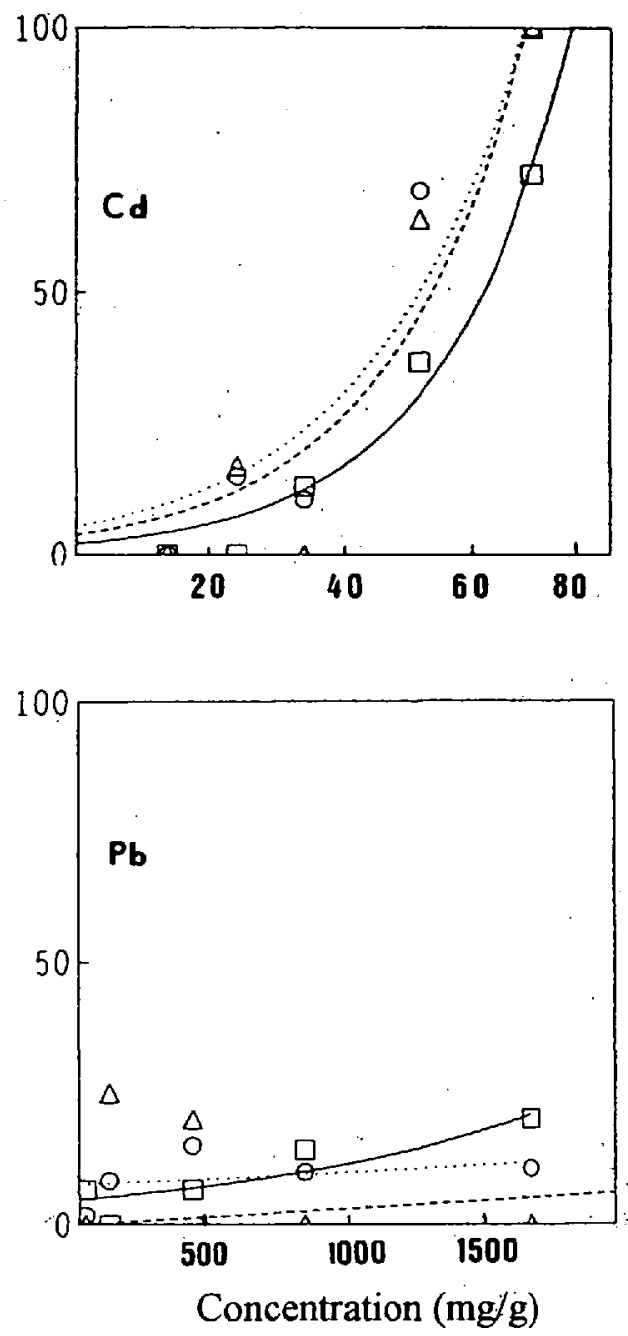

population totale.

Fig. 1. Mortalités (en \%) d'E. variatus observées après 72 heures en présence de concentrations croissantes en $\mathrm{Cd}, \mathrm{Cu}, \mathrm{Pb}$ et $\mathrm{Zn}$.

Fig. 1. \% mortality of $E$. variatus after $72 \mathrm{~h}$-exposure to high concentrations of $\mathrm{Cd}, \mathrm{Cu}, \mathrm{Pb}$ and $\mathrm{Zn}$. 
Excepté avec le cuivre, le seuil de concentration qui entraîne une mortalité globale significative diminue avec le temps (Tableau 3). Ces seuils sont du même ordre de grandeur pour le cuivre et pour le cadmium; seul le zinc entraîne une mortalité aux plus faibles concentrations testées $(11,5 \mathrm{mg} / \mathrm{g})$.

Tableau 3. Concentrations seuils (en $\mathrm{mg} / \mathrm{g}$ de poids sec) entraînant une mortalité significative pour $E$. variatus.

Table 3. Threshold concentrations (in $\mathrm{mg} / \mathrm{g}$ dry weight) producing a significant mortality in $E$, variatus.

\begin{tabular}{llllc}
\hline Métal & cd & Zn & Cu & Pb \\
$24 h$ & 55,2 & 22,9 & 56,7 & $/$ \\
$48 \mathrm{~h}$ & 55,2 & 11,5 & 56,7 & 1600 \\
$72 \mathrm{~h}$ & 25,7 & 11,5 & 56,7 & 400,7 \\
\hline
\end{tabular}

Les mortalités les plus fortes se produisent dans les premières 24 heures (Fig. 2) :

- 55,1 à $84,4 \%$ de la mortalité totale pour le cadmium $(\bar{x}=71,5 \%)$;

- 25,6 à 87,3 pour le cuivre $(\mathrm{x}=69,1 \%)$;

- 24,9 à 92,8 \% dans le cas du zinc $(\mathrm{x}=75,1)$.

Malgré des teneurs élevées, les sels de plomb ne provoquent aucune mortalité significative pendant cette période ; les faibles mortalités observées se produisent au cours des $\mathbf{4 8}$ heures suivantes.

Comme pour les adultes, quelle que soit la durée du test, la mortalité des fragments augmente avec la concentration en cadmium, en zinc et en cuivre et le sel de plomb utilisé n'a aucune influence sur la mortalité des diverses fractions de la population. Pour des concentrations équivalentes de métal, le taux de mortalité des adultes est plus faible que celui des jeunes (Fig. 1).

\section{La $D L-50$}

Les DL-50 calculées pour les jeunes et les fragments sont plus basses que celles calculées pour les adultes (Tableau 4). La sensibilité semble plus liée à la taille des individus qu'à l'âge. La plus grande différence entre la DL-50 des juvéniles et des adultes s'observe pour le zinc, la plus petite pour le cadmium.

\section{Fragmentation}

Dans nos expériences, les métaux n'influencent pas le nombre de fragments issus d'un individu, préalablement observé en élevage par Bouguenec \& Giani (1989). Le taux de fragmentation des adultes diminue globalement, mais de façon très irrégu-
Tableau 4. DL-50 pour le cadmium, le cuivre et le zinc calculées à 24,48 et 72 heures pour $E$. variatus.

Table 4. LD 50 for cadmium, copper and zinc calculated at 24 , 48 and 72 hours for $E$. variatus.

\begin{tabular}{lllll}
\hline Heures & 24 & 48 & 72 \\
\hline \multirow{3}{*}{ Cd } & Adultes & 69,5 & 60,1 & 59,4 \\
& Fragments & 49,3 & 63,5 & 50,4 \\
& Population totale & 55,4 & 58,7 & 49,6 \\
& & 74,8 & 69,6 & 63,4 \\
Cu & Adultes & 71,6 & 34,9 & 65,7 \\
& Pragments & 72,3 & 85,3 & 60,8 \\
& & & \\
Zn & Adultes & 46,2 & 39,7 & 39,7 \\
& Fragments & 28,0 & 16,7 & 17,3 \\
& Population totale & 30,1 & 17,7 & 16,6 \\
\hline
\end{tabular}

lière (Fig. 3), lorsque la concentration de chacun des 4 métaux augmente. Les concentrations moyennes en $\mathrm{Zn}$ augmentent le taux de fragmentation, contrairement aux plus fortes qui l'inhibent presque totalement (Fig. $4 \mathrm{Zn}$ ). En présence de plomb, quelle que soit la concentration, le taux de fragmentation des adultes est très faible au cours des premières 24 heures (Fig. $4 \mathrm{~Pb}$ ) et il augmente ensuite.

\section{Croissance des populations}

Lors des périodes de 72 heures, la croissance de la population résulte de la seule reproduction asexuée par fragmentation. Le cadmium provoque une décroissance importante de la population (voire la disparition totale pour les plus fortes concentrations) à la fois par diminution du taux de fragmentation et augmentation de la mortalité (Fig. $5 \mathrm{Cd}$ ). Il en est de même pour le zinc bien que les concentrations moyennes déterminent une augmentation de la fragmentation des adultes : celle-ci ne compense pas la mortalité. Pour les concentrations basses ou moyennes en cuivre, le taux de fragmentation contrebalance la mortalité et la population stagne. Le plomb diminue le taux de fragmentation et entraîne une décroissance rapide des effectifs, suivie généralement d'une stabilisation de la population (Fig. $5 \mathrm{~Pb}$ ).

\subsection{Effets à moyen terme}

Les effets à plus long terme que peuvent produire séparément le plomb et le cadmium ajoutés à faibles doses mais à intervalles réguliers, sur la croissance d'une population d'E. variatus, ont été étudiés. 
$\mathrm{Cd}$

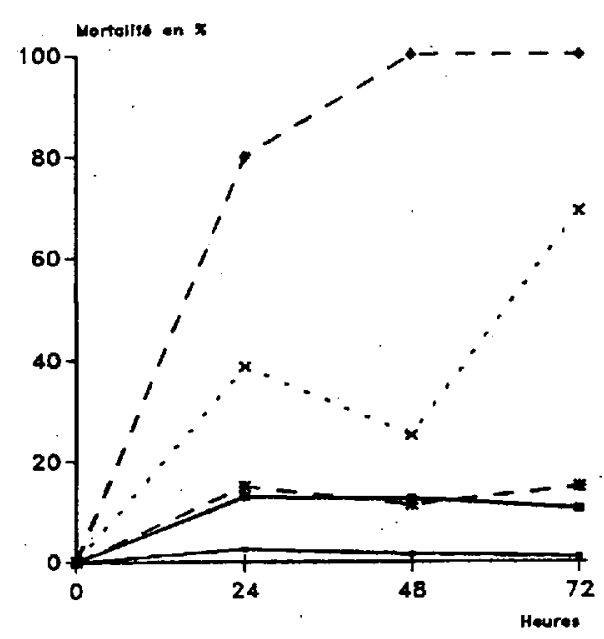

$-0 \mathrm{mg} / \mathrm{g}+14.7 \mathrm{mg} / \mathrm{g} * 25.7 \mathrm{mg} / \mathrm{g}$

$-36.7 \mathrm{mg} / \mathrm{g} * 55,2 \mathrm{mg} / \mathrm{g} * 73,6 \mathrm{mg} / \mathrm{g}$

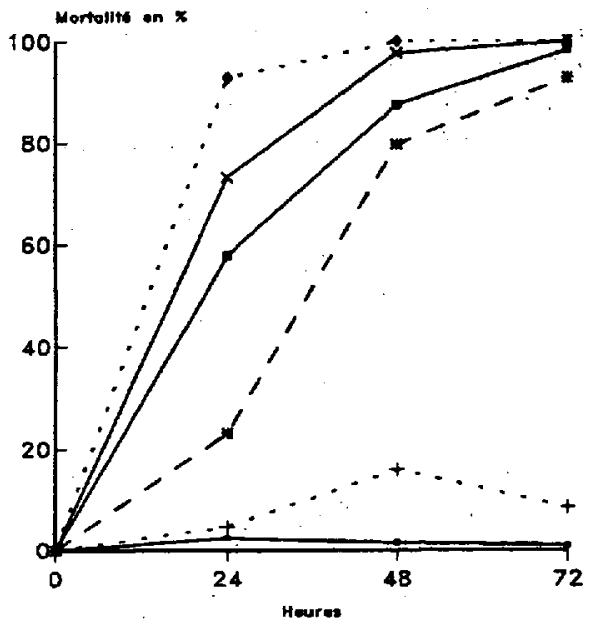

$-0 \mathrm{mg} / \mathrm{g}+11.5 \mathrm{mg} / \mathrm{g} * 22.9 \mathrm{mg} / \mathrm{g}$

$-34,4 \mathrm{mg} / \mathrm{g} * 45,8 \mathrm{mg} / \mathrm{g} * 57,3 \mathrm{mg} / \mathrm{g}$
$\mathrm{CU}$

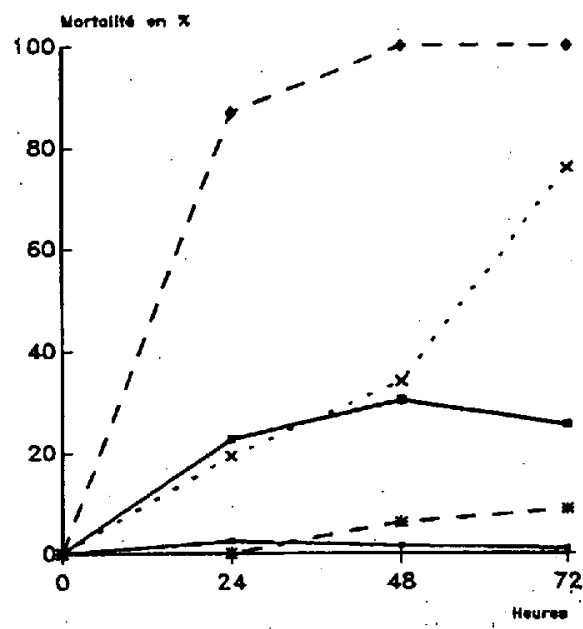

$$
-0 \mathrm{mg} / \mathrm{g}+11,3 \mathrm{mg} / \mathrm{g} * 34 \mathrm{mg} / \mathrm{g}
$$$$
-56.7 \mathrm{mg} / \mathrm{g} * 70,8 \mathrm{mg} / \mathrm{g} \rightarrow 85 \mathrm{mg} / \mathrm{g}
$$

$\mathrm{Pb}$

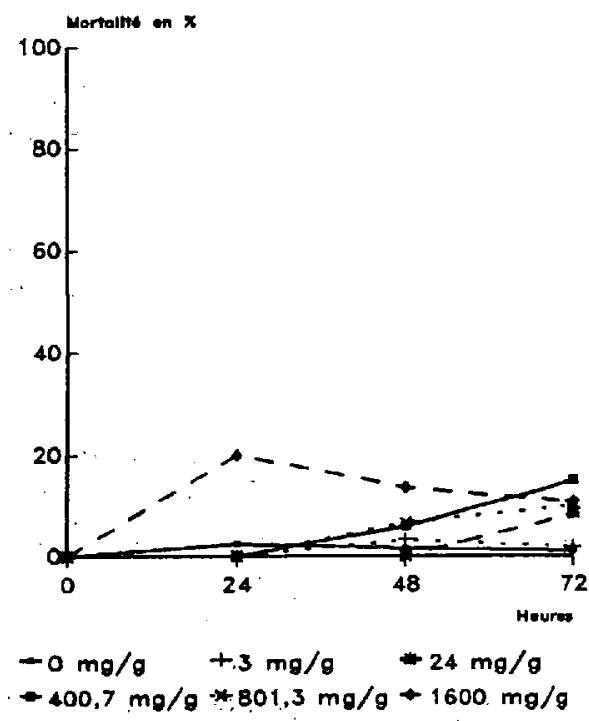

Fig. 2. Evolution de la mortalité d' $E$. variatus en fonction du temps pour différentes concentrations en $\mathrm{Cd}, \mathrm{Cu}, \mathrm{Pb}$ et $\mathrm{Zn}$.

Fig. 2. Temporal changes in the mortality of $E$. variatus for different concentrations of $\mathrm{Cd}, \mathrm{Cu}, \mathrm{Pb}$ and $\mathrm{Zn}$. 


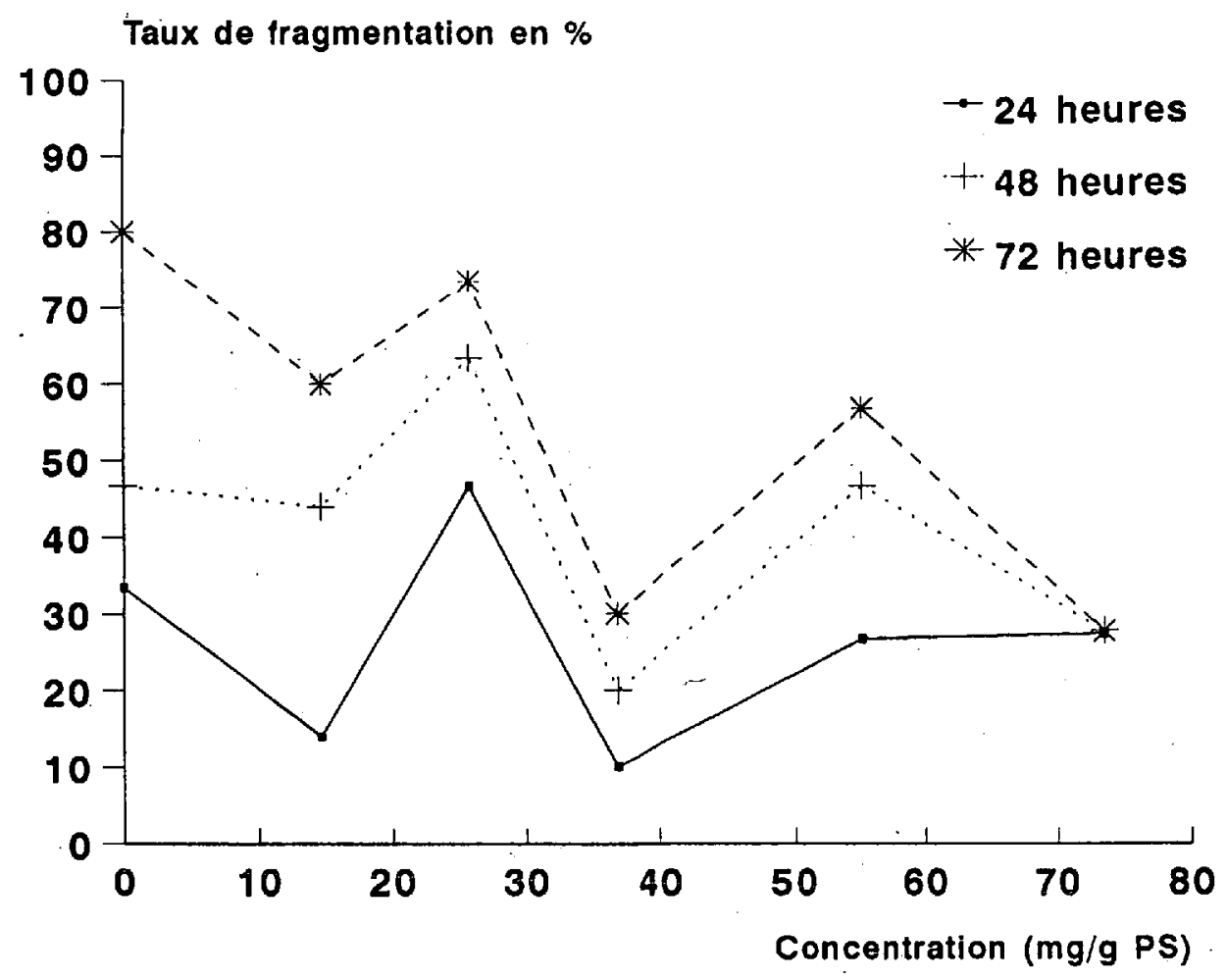

Fig. 3. Variation du taux de fragmentation d' $E$. variatus observé après 24,48 et 72 heures en fonction de la concentration en métal : exemple du cadmium.

Fig. 3. Variation in the fragmentation rate of $E$. variatus after 24,48 and 72 hours as a function of metal concentration : cadmium as the example.

Pour des concentrations en cadmium de $0,3 \mathrm{mg} / \mathrm{g}$ PS et en plomb de $1,2 \mathrm{mg} / \mathrm{g}$ PS, l'évolution des populations est identique à celle du témoin durant les 7 premiers jours. Au-delà, en présence de plomb, la biomasse augmente légèrement alors qu'en présence de Cd toute la population disparaît au cours de la $2^{e}$ semaine (Fig. 6A). Pour des concentrations plus faibles, l'évolution de la biomasse du témoin et celle des populations mises en présence de métaux sont identiques pendant 25 jours (Fig. 6B). En présence de cadmium la biomasse de la population diminue significativement au cours de la sixième semaine. A ce moment là, du fait des apports réguliers en métaux, $3,5 \mathrm{mg} / \mathrm{g}$ PS de plomb et $0,7 \mathrm{mg} / \mathrm{g}$ PS de cadmium ont été introduits dans le milieu.

\subsection{Effets des associations de métaux}

L'action de l'association deux par deux des métaux à des concentrations variables ( 24 combinaisons testées, Tableau 2) a été comparée à l'action individuelle de chacun de ces métaux pour des concentrations identiques.

\section{Effets sur la mortalité}

L'association de métaux peut :

- augmenter significativement le taux de mortalité totale (Fig. 7C) par rapport à celui obtenu par addition des effets des deux métaux pris séparément (synergie additive). C'est le cas lors de l'association du plomb avec du cadmium à forte concentration, de cadmium avec du zinc lorsque les teneurs en zinc sont élevées ou de l'association cuivre-cadmium ;

- n'entraîner aucune modification significative de la mortalité totale (Fig. 7A). Les mélanges plombcuivre, cuivre-zinc, cadmium-zinc et plombcadmium à faible concentration n'entraînent aucun effet de synergie.

- abaisser le taux de mortalité totale (Fig. 7B) ; c'est le cas pour le mélange plomb/zinc, surtout pour les fortes concentrations de zinc. 


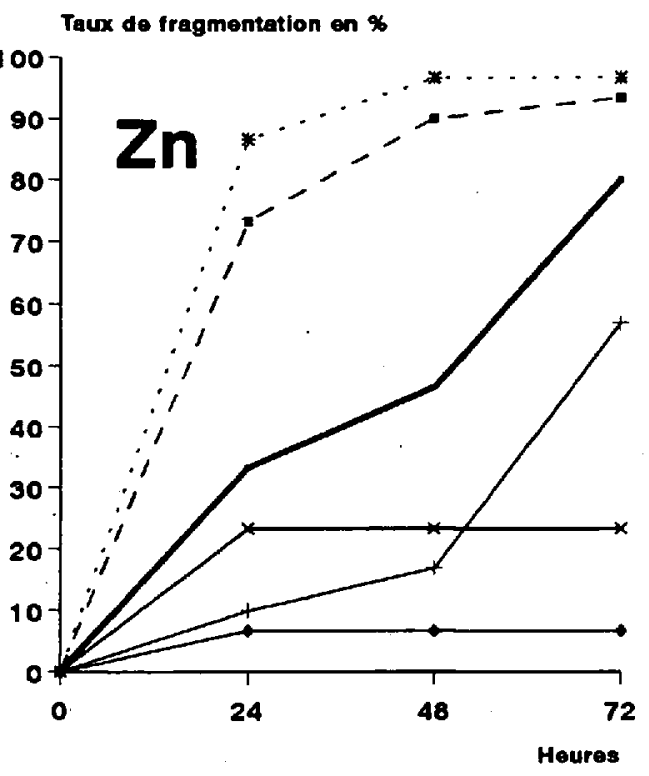

$$
\begin{aligned}
& -0 \mathrm{mg} / \mathrm{g}+11,5 \mathrm{mg} / \mathrm{g} * 22,9 \mathrm{mg} / \mathrm{g} \\
& --34,4 \mathrm{mg} / \mathrm{g} * 45,8 \mathrm{mg} / \mathrm{g}+57,3 \mathrm{mg} / \mathrm{g}
\end{aligned}
$$

Taux de fragmentation on $\%$

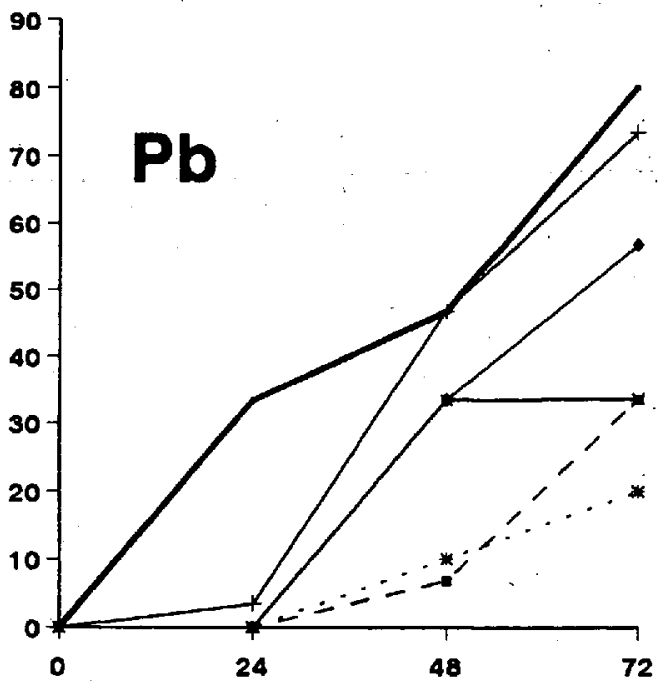

Heures

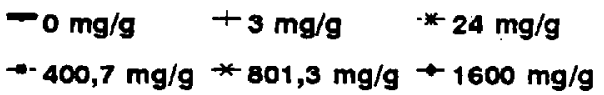

Fig. 4. Evolution dans le temps du taux de fragmentation d'E. variatus en fonction de la concentration en zinc $(\mathrm{Zn})$ et en plomb $(\mathrm{Pb})$.

Fig. 4. Temporal changes in the fragmentation rate of $E$. variatus as a function of the concentration of zinc $(\mathrm{Zn})$ and lead $(\mathrm{Pb})$.
Teux de crolaance en $\%$

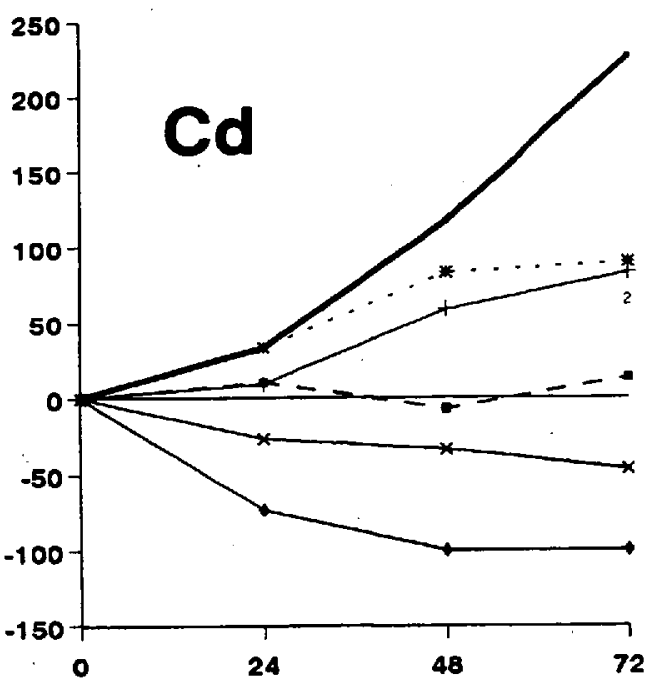

Heures

$$
\begin{aligned}
& -0 \mathrm{mg} / \mathrm{g}+14,7 \mathrm{mg} / \mathrm{g} * 25,7 \mathrm{mg} / \mathrm{g} \\
& -36,7 \mathrm{mg} / \mathrm{g} * 55,2 \mathrm{mg} / \mathrm{g} \rightarrow 73,6 \mathrm{mg} / \mathrm{g}
\end{aligned}
$$

\section{Taux de crolesance on $\%$}

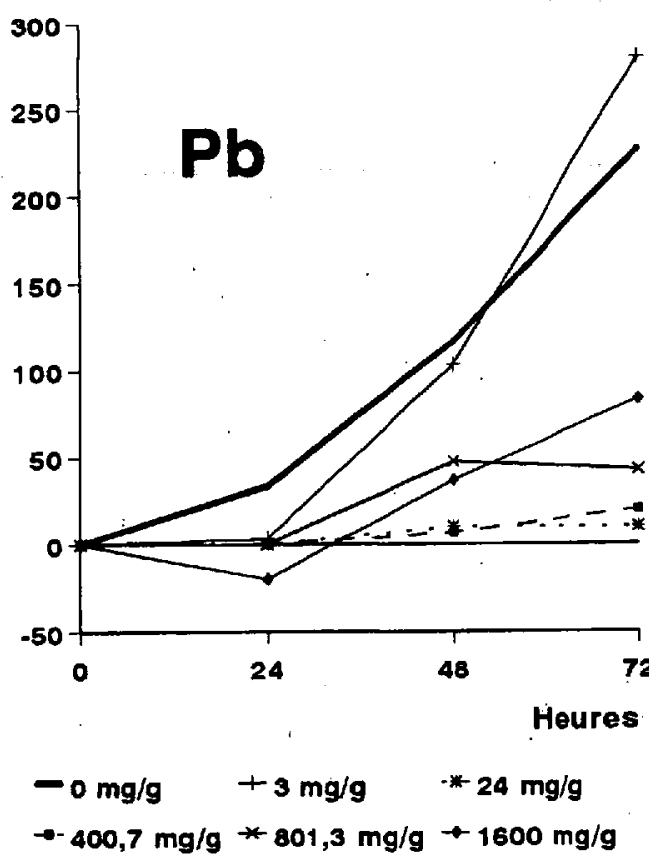

Fig. 5. Evolution dans le temps du taux de croissance de la population d' $E$. variatus en fonction de la concentration en cadmium $(\mathrm{Cd})$ et en plomb $(\mathrm{Pb})$.

Fig. 5. Temporal changes in the growth rate of the population of $E$. variatus as a function of the concentration of cadmium (Cd) and lead (Pb). 

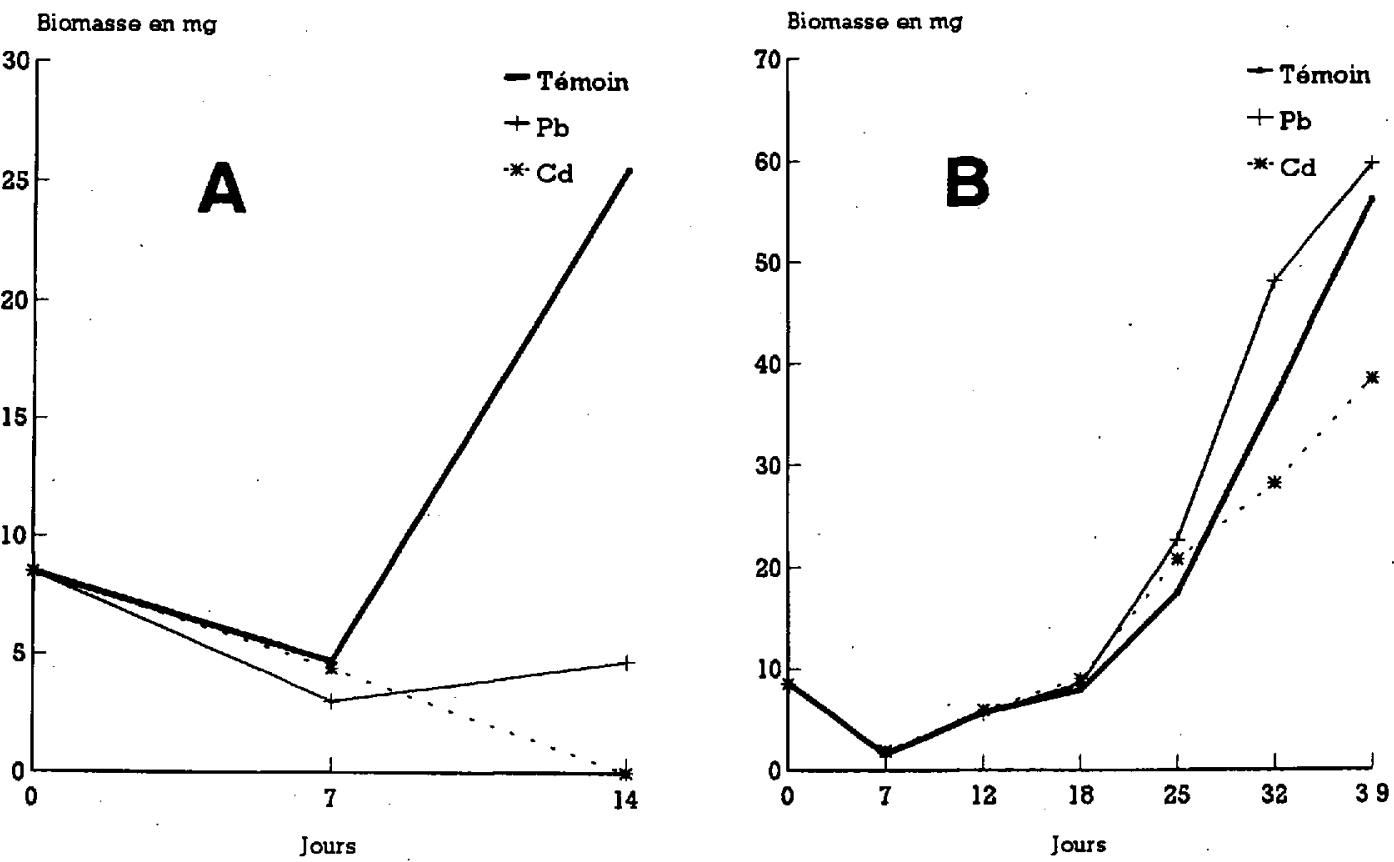

Fig. 6. Evolution dans le temps de la biomasse de populations d'E. variatus en présence d'un apport régulier de $\mathrm{Pb}$ ou de Cd comparé à celui d'une population témoin. A. - Pb: $1,2 \mathrm{mg} / \mathrm{g} \mathrm{PS} ; \mathrm{Cd}: 0,3 \mathrm{mg} / \mathrm{g} \mathrm{PS}$. B. - Pb:0,5 mg/g PS; Cd : 0,1 mg/g PS.

Fig. 6. Temporal changes in the biomass of populations of $E$. variatus in the presence of a regular application of $\mathrm{Pb}$ or $\mathrm{Cd}$ compared to that of a control population. A. $-\mathrm{Pb}: 1.2 \mathrm{mg} / \mathrm{g}$ dry weight ; $\mathrm{Cd}: 0.3 \mathrm{mg} / \mathrm{g}$ dry weight. $\mathrm{B} .-\mathrm{Pb}: 0.5 \mathrm{mg} / \mathrm{g}$ dry weight ; $\mathrm{Cd}: 0.1 \mathrm{mg} / \mathrm{g}$ dry weight.

$\mathrm{Cu} 34.00 \mathrm{Zn} 22.9$

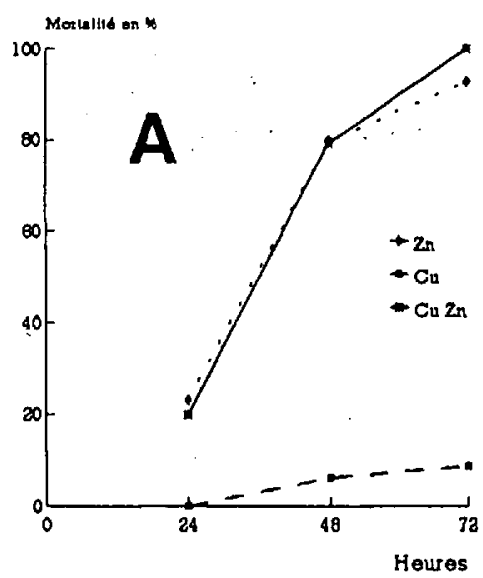

$\mathrm{Pb} 24.00 \mathrm{Zn} 22.9$

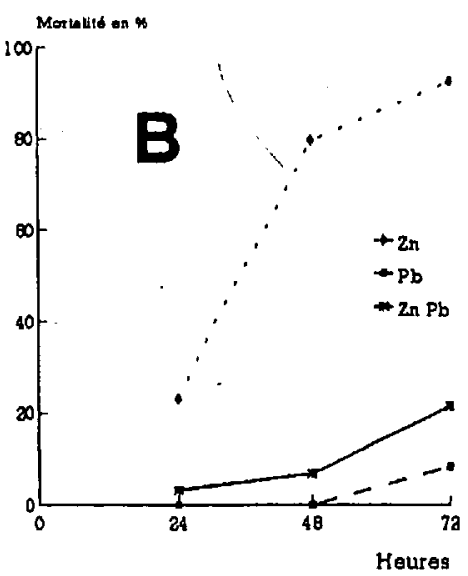

$\mathrm{Cu} 34.00 \mathrm{Cd} 36.7$

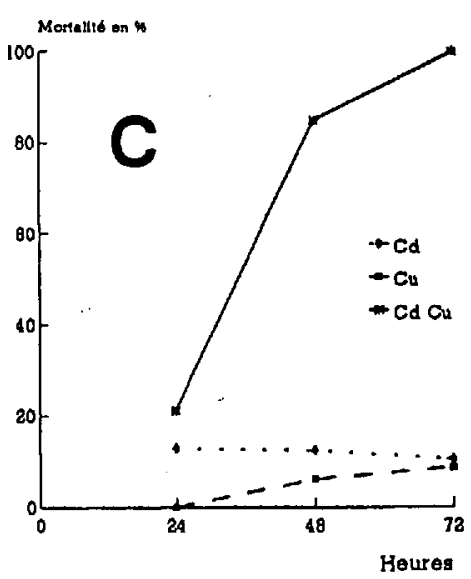

Fig. 7. Mortalité d' $E$. variatus en présence d'un mélange de sels de deux métaux comparée à celle obtenue par l'action isolée de chacun dés sèls de ces mêmes métaux. Exemples des effets observés: A : pas d'effet ; B : effet antagoniste ; C : synergie.

Fig. 7. Mortality of $E$. variatus in the presence of a mixture of salts of two metals compared to that obtained with the separate action of each of the salts of the same metals. Examples of different effects : A : no effet ; B : antagonistic effect ; C : synergy. 


\section{Effets sur la fragmentation}

On observe une hausse du taux de fragmentation avec les mélanges plomb/cuivre. Les autres associations (plomb/zinc, plomb/cadmium, cadmium/ zinc, cuivre/cadmium et cuivre/zinc) provoquent des taux de fragmentation inférieurs à la somme des effets des 2 métaux pris séparément (Tab. 5).

\subsection{Influence des facteurs environnementaux}

La température n'a pas de conséquences sur la dynamique des populations étudiées excepté pour le cadmium dont la toxicité à $30^{\circ} \mathrm{C}$ s'est avérée beaucoup plus forte qu'à 10 ou $20^{\circ} \mathrm{C}$ (Fig. 8A).

C'est pour les plus faibles teneurs $(13,5 \%)$ en matière organique des sédiments que la toxicité des métaux est la plus forte ; l'action des métaux diminue lorsque la teneur en matière organique augmente et dans le cas du cadmium la relation est même linéaire (Fig. 8B).
Les valeurs de $\mathrm{pH}$ testées ne semblent pas avoir d'influence significative sur l'action du plomb et du cadmium vis-à-vis d' $E$. variatus : l'évolution du nombre d'individus est parallèle à celle du témoin (Fig. 8C); les plus faibles valeurs traduisent seulement la mortalité inhérente à la présence des métaux.

\section{Discussion}

Les métaux lourds ont des effets variables selon l'espèce animale considérée (Sloff 1983). Par exemple, le cuivre est plus toxique que le zinc pour Nais sp. (Rehwoltd et al. 1973) et pour plusieurs espèces de Polychètes (Reish \& Carr 1978, Reish \& Gerlinger 1984, Reish 1985). Pour $E$. variatus, quelles que soient la durée de l'expérience et la fraction de population considérée, le zinc est le plus toxique des quatre métaux testés, suivi par le cadmium puis le cuivre.

Tableau 5. Taux de fragmentation mesuré et théorique (en \%) d'E. variatus après 24 h en présence d'une association de métaux. Taux théorique $=$ somme des taux de fragmentation pour chacun des métaux considérés isolément. Pour la nature des associations, voir la signification des codes dans le tableau 2.

Table 5. Measured and theoretical (in \%) fragmentation rate of E. variatus after $24 \mathrm{~h}$ exposure to a metals association. Theoretical rate $=$ sum of the fragmentation rates for each of the metals considered singly. See the codes in Table 2 for the different associations.

\begin{tabular}{|c|c|c|c|c|c|}
\hline Association & $\mathrm{Pb} / \mathrm{Cu}$ & CP1 & $\mathrm{CP} 2$ & CP3 & CP4 \\
\hline Taux & $\begin{array}{l}\text { Mesuré } \\
\text { Théorique }\end{array}$ & $\begin{array}{l}6,7 \\
3,4\end{array}$ & $\begin{array}{r}10,1 \\
6,8\end{array}$ & $\begin{array}{c}13,4 \\
0\end{array}$ & $\begin{array}{r}10,1 \\
3,4\end{array}$ \\
\hline Association & $\mathrm{Pb} / \mathrm{Zn}$ & PZ1 & PZ2 & P23 & PZ4 \\
\hline Taux & $\begin{array}{l}\text { Mesuré } \\
\text { Théorique }\end{array}$ & $\begin{array}{c}0 \\
13,4\end{array}$ & $\begin{array}{l}10 \\
90,1\end{array}$ & $10^{3,4}$ & $\begin{array}{l}20 \\
86,7\end{array}$ \\
\hline Association & $\mathrm{Cu} / \mathrm{Cd}$ & $\operatorname{cc} 1$ & $\operatorname{cc} 2$ & $\operatorname{cc} 3$ & $\mathrm{CC} 4$ \\
\hline Taux & $\begin{array}{l}\text { Mesuré } \\
\text { Théorique }\end{array}$ & $14^{6,7}$ & $\begin{array}{r}6,7 \\
10,1\end{array}$ & $\begin{array}{r}6,7 \\
17,4\end{array}$ & $\begin{array}{r}3,4 \\
13,5\end{array}$ \\
\hline Association & $\mathrm{Cu} / \mathrm{zn}$ & $\mathrm{zC} 1$ & $\mathrm{zC} 2$ & $\mathrm{zc} 3$ & $\mathrm{zC4}$ \\
\hline Taux & $\begin{array}{l}\text { Mesuré } \\
\text { Théorique }\end{array}$ & $\begin{array}{l}16,7 \\
10\end{array}$ & $\begin{array}{r}6,7 \\
13,4\end{array}$ & $\begin{array}{l}33,4 \\
86,7\end{array}$ & $\begin{array}{l}43,4 \\
90,1\end{array}$ \\
\hline Association & $\mathrm{Cd} / \mathrm{zn}$ & CZ1 & $\mathrm{cz2}$ & $\mathrm{Cz} 3$ & $\mathrm{CZ4}$ \\
\hline Taux & $\begin{array}{l}\text { Mesuré } \\
\text { Théorique }\end{array}$ & $\begin{array}{l}20 \\
24\end{array}$ & $\begin{array}{r}13,4 \\
20,1\end{array}$ & $\begin{array}{r}60 \\
100\end{array}$ & $\begin{array}{l}13,4 \\
96,8\end{array}$ \\
\hline
\end{tabular}



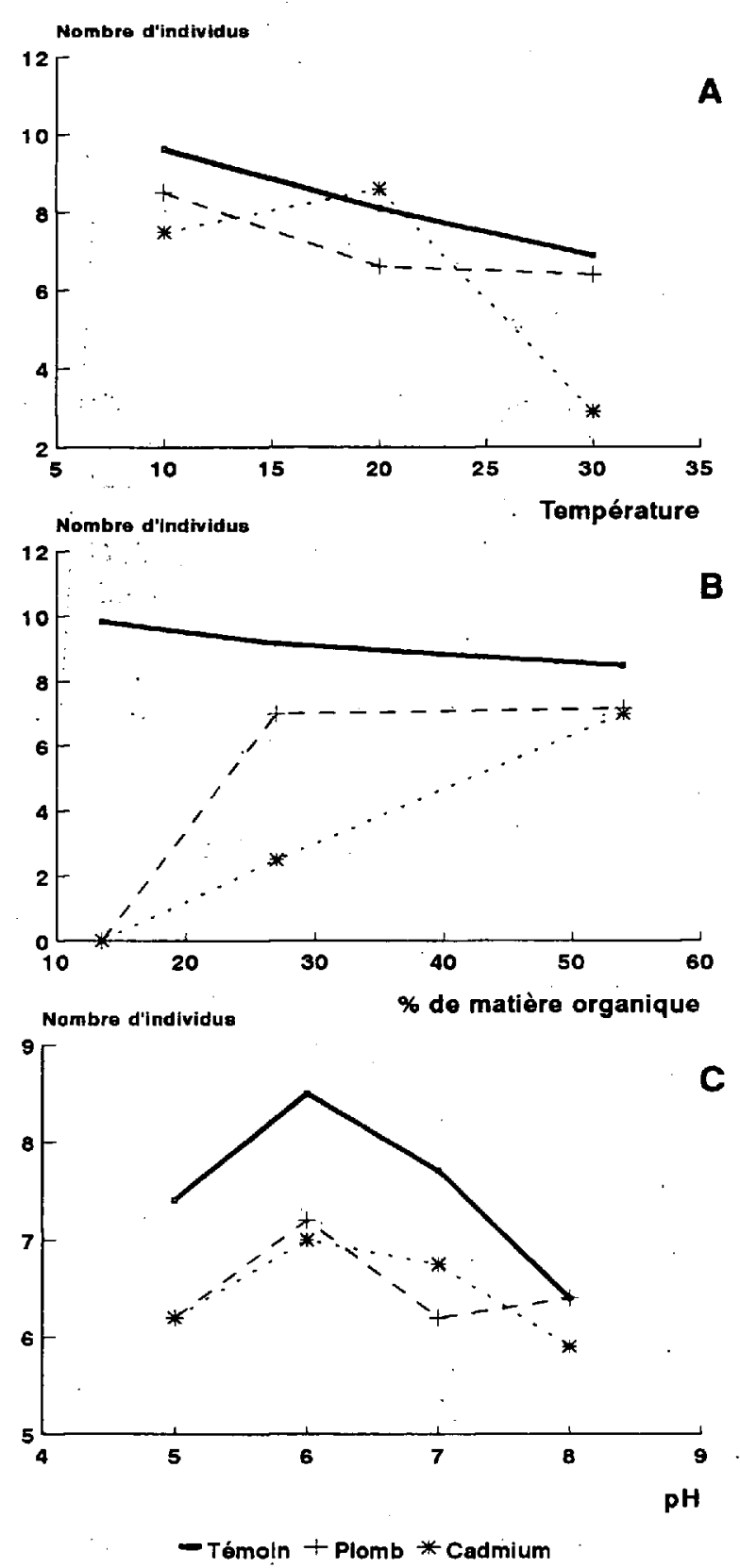

Fig. 8. Influence de la température (A), de la teneur en matière organique (B) et du pH (C) du sédiment sur la toxicité du cadmium et du plomb pour $E$. variatus, exprimée par le nombre de vers vivants au bout de $72 \mathrm{~h}$.

Fig. 8. Influence of temperature (A), of organic matter content (B) and of $\mathrm{pH}(\mathrm{C})$ of the sediment on the toxicity of cadmium and lead for $E$. variatus, expressed as the number of worms still living after $72 \mathrm{~h}$.
Le plomb est, parmi les métaux lourds couramment testés, le métal le moins toxique à court terme (Biesinger \& Christensen 1972, Reish \& Carr 1978, Reish 1985). Nos expériences n'ont pas permis de mettre en évidence d'effet significatif du citrate et du carbonate de plomb; sur la mortalité, à court terme, d'E. variatus. Ces sels ont un produit de solubilité très faible et se dissocient peu dans l'eau. Nous avons donc testé le nitrate de plomb $\left(\mathrm{Pb}(\mathrm{NO} 3)_{2}\right)$ dont le produit de solubilité est plus élevé et qui a été choisi par Jones (1938) pour évaluer la sensibilité au plomb de plusieurs espèces aquatiques. Sous cette forme le plomb présente une toxicité aiguë pour E. variatus.

L'effet des métaux lourds varie aussi en fonction des conditions de milieu : leur nocivité peut dépendre de la température, du $\mathrm{pH}$, de la dureté de l'eau ou de la salinité (Mathis \& Cumming 1973, Chapmann \& Brinkhurst 1984). La toxicité des métaux utilisés dans ce travail a peu varié avec la température sauf pour le cadmium qui s'est avéré plus toxique à $30^{\circ} \mathrm{C}$. Elle s'est révélée indépendante du $\mathrm{pH}$, dans la gamme des valeurs testées (entre pH 5 et 8) avec $E$. variatus qui correspond à celle où une espèce voisine, $E$. albidus, ne manifeste aucun signe de gêne (Römbke \& Knacker 1989). Par contre la toxicité a diminué avec l'augmentation de la teneur en matière organique du substrat. Les métaux lourds de l'eau interstitielle sont fixés ou complexés par la matière organique et leur toxicité, essentiellement dûe aux ions métalliques libres, diminue (Nebeker et al. 1986, Hamilton et al. 1987). Les conditions de milieu d'un éventuel test utilisant $E$. variatus amoindriront ce problème car les sédiments aquatiques ont une teneur en matière organique beaucoup plus faible que le terreau utilisé dans nos essais. Subsistera l'inconvénient d'utiliser un sédiment partiellement déshydraté (pas d'eau surnageante) qui atténuera la toxicité des métaux par rapport aux sédiments in situ: la majorité des toxiques sont 2000 fois moins toxiques dans le sol que dans l'eau (Römbke \& Knacker 1989).

Parmi les Oligochètes, les DL-50 observées pour un même métal sont très variables : pour le cadmium elles vont de $3,5 \mathrm{mg} / 1$ à $12,9 \mathrm{mg} / 1$ pour diverses espèces de Tubificidae (Chapman \& Brinkhurst 1984). De même la toxicité du sulfate de cuivre varie pour des espèces aussi proches que Nais communis et N. variabilis (Learner \& Edwards 1963). Si l'on compare les DL-50 observées, il semble qu' $E$. variatus 
soit, par exemple, beaucoup plus résistant au cadmium que $L$. hoffmeisteri ou Tubifex tubifex, 2 espèces aquatiques étudiées par Chapman \& Brinkhurst (1984). Mais les différences peuvent aussi résulter de différences de toxicité en fonction des conditions expérimentales, telle celle que nous avons observée pour le plomb. Les DL-50 obtenues pour le cuivre avec cette espèce sont 100 à 300 fois plus élevées que celles pour Nais sp. et 3 fois plus élevées pour le cadmium ; les DL-50 pour le zinc sont équivalentes. On peut donc conclure qu' $E$. variatus est une espèce peu ou moyennement sensible.

Dans le cas d'E. variatus, le zinc, le cadmium et le cuivre provoquent une mortalité significative des adultes et des fragments. Le taux de mortalité des adultes est plus faible que celui des jeunes et des fragments : la sensibilité varie donc selon les stades et semble liée à la taille. La mortalité est généralement un paramètre peu sensible (Dean-Ross 1983, Wiederholm 1987) et c'est pour cela que plusieurs auteurs ont proposé d'utiliser des paramètres sublétaux portant notamment sur le taux de reproduction (Wiederholm 1987, Westheide \& BethkeBeilfuss 1991). Chez $E$. variatus, la toxicité se manifeste aussi par certains effets sublétaux, par exemple sur la fragmentation. Les métaux, y compris le plomb qui n'a pas d'effet sur la mortalité, entraînent généralement une baisse du taux de fragmentation des adultes dans les premières 24 heures; celle-ci est d'autant plus forte que la concentration augmente mais le nombre de fragments que donne un individu est indépendant de la présence des métaux. Notons que l'augmentation importante du taux de fragmentation observée pour des concentrations moyennes en zinc et en cuivre semble pouvoir être interprétée comme une réaction ultime des individus avant la mort. Le phénomène de fragmentation, peu courant chez les Enchytraeidae, pose des problèmes dans le déroulement des tests et complique l'interprétation des résultats. Il semble très diffícile d'établir un critère prenant en compte la fragmentation comme l'avaient déjà noté Dermott \& Medawar (1992) avec Lumbriculus variegatus.

Cette étude met en évidence certaines caractéristiques qui rendent difficile l'utilisation de cette espèce dans un biotest, contrairement à une espèce jumelle ( $E$. crypticus Westheide et Graefe, 1992). Certains paramètres relatifs à la reproduction sexuée ont fait de cette dernière espèce un organisme test intéressant (Westheide \& Bethke-Beilfuss 1991).

\section{Références}

Bethke-Beilfuss D. 1986. - Auswirkungen von Pestiziden auf den. Lebenszyklus verschieener Enchytoeus-Arten (Oligochaeta, Enchytraeidae). Thèse de Doctorat, University of Osnabrück, R.F.A., 101 p.

Biesinger K.E. \& Christensen G.M. 1972. - Effects of various metals on survival, growth, reproduction and metabolism of Daphnia magna. J. Fish. Res. Board. Can., 29 (12) : 1691-1700.

Bouguenec V. 1987. - L'élevage en masse d'Enchytraeidae : étude bibliographique, mise au point expérimentale et tests dans l'alimentation des poissons. Thèse de Doctorat, Université Paul Sabatier, Toulouse, France, 352 p.

Bouguenec V. \& Giani N. 1989. - Biological studies upon Enchytraeus variatus Bouguenec et Giani, 1987 in breeding cultures. Hydrobiologia, $180: 151-165$.

Chapman P.M. \& Brinkhurst R.O. 1984. - Lethal and sublethal tolerance of aquatic oligochaetes with reference to their use as a biotic index of pollution. Hydrobiologia, 115 : 139-144.

Dean-Ross D. 1983. - Methods for the assessment of the toxicity of environmental chemicals to earthworms. Regulatory Toxic Pharmac., 3 : 48-59.

Dermott R. \& Munawar M. 1992. - A simple and sensitive assay for evaluation of sediment toxicity using Lumbriculus variegatus (Müller) ; Hydrobiologia 235/236 : 407-414.

Hamilton R.D., Klaverkamp J.F., Lockhart W.L. \& Wagemann R. 1987. - Major aquatic contaminants, their sources, distribution and effects. Bull. Fish. Aquat. Sci. Can., 215 : 357-386.

Jones J.R.E. 1938. - The relative sensitivity of aquatic species to lead in solution. J. Anim. Ecol., 7 : 287-289.

Kaufman E.S. 1975. - Certain problems of Phenol intoxication of Enchytraeus albidus from the viewpoint of stress. Hydrobiol J., $11:$ :44-46.

Learner M.A. \& Edwards R.W. 1963. - The toxicity of some substances to Nais (Oligochaeta). Proc. Soc. Wat. Treat. Exam., 12 : 161-168.

Mathis B.J. \& Cummings T.F. 1973. - Selected metals in sediment water and biota in the Illinois River. J. Wat. Pollut. Control. Fed., 45 : 1573-1583. :

Nebeker A.V., Onjukka S.T., Caims M.A., Frawczyk D.F. 1986. - Survival of Daphnia magna and Hyalella azteca in cadmium spikes water and sediment. Environ. Toxicol. Chem., 5 (10) : $933-938$.

Reish D.J. 1985. - The use of the polychaetous annelid Neanthes arenaceodentata as a laboratory experimental animal. Thetys, 11 (3-4) : 335-341.

Reish D.J. \& Carr R.S. 1978. - The effect of heavy metals on the survival, reproduction, development and life cycles for two species of polychaetous annelids. Mar. Pollut, Bull., 9 : 24-27.

Reish D.J. \& Gerlinger T.V. 1984. - The effects of cadmium, lead and zinc on survival and reproduction of the polychaetous annelid Neanthes arenaceodentata. Proceeding of the first International Polychaete Conference, Linnean Society of new South Wales, Sydney, Australia, Hutchings, P.A., ed. : 383-389. 
Rehwoldt R., Lasko L., Shaw C. \& Wirhowski E. 1973. - The acute toxicity of some heavy metal ions toward benthic organisms. Bull. Environ. Contam. Toxicol., 10 (5) : 291-294.

Römbke J. \& Knacker T. 1989. - Aquatic toxicity test for enchytraeids. Hydrobiologia, $180: 235-242$.

Sloof W. 1983. - Benthic macroinvertebrates and water quality assessment : some toxicological considerations. Aquatic Toxicol., $491: 73-82$.
Wiederholm T, 1987. - Bulk sediment bioassays with five species of freshwater oligochaetes. Wat. Air Soil Pollut., 36 : 131-154.

Westheide W. \& Bethke-Beilfuss D. 1991. - The sublethal enchytraeid test system : guideliness and some results. In Esser E. \& Overdieck D. (eds), Modern Ecology : Basic and applied aspects. Elsevier, Amsterdam, 497-508. 